\section{Change in Insurance Status and Cost-related Medication Non-adherence among Older US Adults with Diabetes from 2010 to 2014}

\section{Abstract}

Objective: Cost-related medication non-adherence (CRN) is a persistent challenge in health care in the U.S. Insurance coverage is a key determinant of access to medical care. We seek to examine the CRN rates among the older diabetes adult population in the U.S. from 2010 to 2014 when the major provisions of the Affordable Care Act came into force.

Research design and method: Data from the 2010 and 2014 Health and Retirement Study (HRS) were used for this study. CRN is identified if a respondent indicated taking less medication than was prescribed because of the cost, while diabetes is self-reported. We assessed the change in CRN rates by insurance status using multivariable logistic regression analysis.

Results: A total of 4,741 and 4,505 diabetes adults aged 50 or older in 2010 and 2014 were included in the analyses, representing 18.8 million and 19.1 million older adults with diabetes respectively. Overall, the percentage of dual-eligible diabetes patients increased from $8 \%$ to $10 \%$ and the uninsured decreased from $6 \%$ to $4 \%$ based on weighted population estimates. The CRN rates decreased from $27 \%$ to $21 \%$ and from $12 \%$ to $10 \%$ for those between 50 and 64 , and 65 or older, respectively from 2010 to 2014. Race (African American) became a less significant factor for variations in CRN rates in $2014(p=0.24)$.

Conclusions: There is an encouraging reduction in CRN rates after implementation of the ACA. However, CRN rates among diabetes patients between 50 and 65 of age remained high.

Keywords: Cost-related medication non-adherence; Affordable care act; Health insurance

\section{Zhang JX ${ }^{1 *}$, Bhaumik D², Huang ES ${ }^{1}$, Meltzer DO ${ }^{1-3}$}

1 Department of Medicine, The University of Chicago, Chicago, USA

2 Harris School of Public Policy, The University of Chicago, Chicago, USA

3 Department of Economics, The University of Chicago, Chicago, USA

\section{*Corresponding author: \\ James X. Zhmang, PhD, MS \\ $\equiv$ xzhang1@medicine.bsd.uchicago.edu}

Department of Medicine, Section of Hospital Medicine, The University of Chicago, Chicago, USA

Tel: $+1-7738341631$

Citation: Zhang JX, Bhaumik D, Huang ES, Meltzer DO (2018) Change in Insurance Status and Cost-related Medication Nonadherence among Older US Adults with Diabetes from 2010 to 2014. J Health Med Econ Vol.5 No.1:7

Received: May 23, 2018; Accepted: September 27, 2018; Published: October 11, 2018

\section{Introduction}

Despite the recent effort to increase insurance coverage and strengthen insurance benefit design through the Affordable Care Act, the cost barrier to health care remains uneven and persistent in the USA [1-3]. Cost-related medication non-adherence (CRN), a behavior where patients take less medication due to costs, has been a focus of policy research in understanding if an increase in insurance coverage through health policy can lead to reduction of access barrier $[4,5]$. Hence, measuring the change in prevalence rates in CRN after the implementation of ACA provides a meaningful gauge of its impact on access barriers. This is important not only to policy impact research per se, but also to the well-being of patients and society, as research has shown that the cost barrier to medications adversely affects patient outcomes and raises overall health care costs to society [6-9].

There is robust discussion in the literature regarding the underuse of medication among diabetes patients, pointing out that economic pressure and belief of medication effectiveness and harm are the most common reasons for underuse, and that cost-related adherence problems are especially common among patients with diabetes with comorbid diseases [10-12]. However, little is known about if the prevalence in CRN has seen any change after the recent expansion of insurance coverage through the ACA as Medicaid expansion made many more older adults with diabetes eligible for Medicaid, regardless of whether they were previously uninsured, or insured through Medicare or private insurance. With additional and more generous coverage 
under Medicaid, the out-of-pocket cost burden borne by patient's decreases, and hence CRN should decrease within this population, an important but often overlooked policy effect of the ACA. However, empirical examination of the ACA and CRN rates is absent from the literature. Moreover, if the CRN rates remain unevenly high, more intervention is needed to address the access barrier.

We therefore propose to evaluate the change in CRN prevalence rates by insurance status between 2010, when the ACA was signed into law, and 2014, when major provisions came into force [13]. We hypothesize a change in insurance status among the diabetes patients as the ACA expanded Medicaid coverage, changing the composition of insurance status within the diabetes population and leading to further changes in prevalence rates among diabetes patients by types of insurance coverage. We aimed to examine such a change across the age stratification of the adult population by between 50 and 64 and 65 or older, as diabetes patients above 65 are inherently different from those below 65 due to work/employment status (i.e., retirement status) and Medicare coverage (including Part $D$ ), and hence a stratified analysis by age strata may illuminate better the role of change in insurance coverage due to policy action for heterogeneous subpopulations.

\section{Research Design and Methods}

\section{Data and cost-related non-adherence to medical care}

Data from the 2010 and 2014 Health and Retirement Study (HRS) were used for this study. The HRS is a longitudinal panel study that surveys a representative sample of approximately 20,000 people in America over the age of 50 about their income, employment, health insurance, physical health, cognitive functioning, and health care expenditures [14]. Data for the survey is collected primarily by telephone interview every 2 years. Since this is a national assessment of prevalence of CRN rates among diabetes patients in 2010 and 2014, the analysis in this study was restricted to survey respondents in 2010 and 2014, respectively, who reported that a physician had told them that they had diabetes in the respective year.

CRN was measured by asking participants, "Sometimes people delay taking medication or filling prescriptions because of the cost. At any time since the last interview or in the last two years have you ended up taking less medication than was prescribed for you because of the cost?" Participants answered either yes or no, although they had the option to refuse to answer or say that they did not know.

\section{Socio-economic strata}

The HRS includes questions about demographics and socioeconomic characteristics, including age, race, ethnicity, education level, ethnicity, and place of residence. We categorized patients into two age groups, 50-64 years and 65 or older. We hypothesized that patients in the age group of 50-64 years old might be at elevated risk of CRN because they may not be eligible for Medicare which could provide low-cost outpatient drug insurance benefits. In addition, diabetes patients with pre-existing conditions under 65 years of age may have found it difficult to purchase individual health insurance due to high out-of-pocket costs even if they are eligible for an individual policy under the ACA. We also categorized the educational attainment at the level of completion of high school education as we hypothesized that educational attainment may play a role in understanding the benefits of medication adherence. Income is included as it is an important enabling factor for purchasing goods and services. Since the elderly may have multiple income sources including pension, retirement, and investment accounts, we created a variable of income by summarizing the respondent's income, including wages and salary, self-employment, business profits, pension, tips, commissions, bonds, and other compensations and benefits including welfare, and veteran benefits. Hence this is a comprehensive measure of income available to the respondent on an annual basis. Since income is highly skewed, we took log transformation of income in the multivariate regression analysis to conform to the normality assumption [15].

\section{Health and comorbid conditions}

We included a number of covariates in our multivariable regression analysis, including the number of limitations in Activities of Daily Life (ADLs), Instrumental Activities of Daily Life (IADLs), and comorbid conditions to control for potential confounding. We included the functional status variable because it may reflect the burden of illness borne by diabetes patients, which may increase costs such as transportation to see the medical provider and costs to obtain prescription or have prescription refilled [16].

We also included a variable of depression. Depressive symptoms were associated with CRN in elderly Medicare enrollees and Medicare enrollees with disabilities [17]. We also included a set of comorbid conditions including heart conditions, stroke, and cancer, as there are established clinical link between diabetes and those comorbid condition although potential biologic links between cancer and diabetes are incompletely understood [18]. These comorbid condition are likely to increase the overall disease burden of diabetes patients, increasing their out-ofpocket payments and hence their CRN rates.

\section{Stratified and multivariate regression analysis}

We hypothesized a difference in CRN by age stratum of between 50 and 64 and 65 or older across insurance status as explained above, and hence calculated prevalence rates of CRN in each age stratum for insurance status. We included 6 insurance statuses in our analysis, by a hierarchical order of MedicareMedicaid dual eligibility, Medicare without Medicaid, Medicaid without Medicare, private insurance without public insurance, no insurance, and other public coverage [19]. Insurance status is complex because patients can have multiple public and private insurance coverage, and such a hierarchical categorization of insurance status allows us to identify patients' coverage by their primary payer.

With the age strata of 50-64 and 65+ mentioned above, we performed chi-square tests to compare the CRN rates across insurance status for the year of 2010 and 2014, respectively. 
We calculated such rates for the sample as well as weighted population estimates. To address potential confounding and overlapped effect by socio-economic variables and health variables, we further conducted a multivariable regression analysis of CRN using insurance variables as predictors, controlling for age, gender, race, ethnicity, income, functional status, and comorbid conditions, combining diabetes patients at all age in this sample. Statistical significance level was set at 0.05 . We reported c statistics for the overall model performance in the multivariate logistic regression analysis [20]. All analyses were performed using Stata 14 software (Stata, College Station, Texas, 2015).

\section{Results}

There were 4,737 respondents who self-identified as having diabetes in the 2014 HRS. We excluded 75 respondents under the age of 50, 69 patients who refused to report or had unknown health insurance information, 18 patients with missing race information, 2 respondents with missing gender information, 30 patients with unknown education status, and 20 individuals who either did not know or refused to answer the question regarding CRN, resulting a final sample of 4,505 patients in the analysis for 2014.

Similarly, there were 5,037 respondents who self-identified as having diabetes in the 2010 HRS. We excluded 157 respondents under the age of 50,37 respondents who refused to report or had unknown health insurance information, 13 respondents with missing race information, 6 respondents with unknown education status, and 61 respondents who either did not know or refused to answer the question regarding CRN who were dropped, resulting a final sample of 4,741 diabetes patients in the analysis for 2010.

Table 1 shows the CRN socio-economic distribution of the respondents for the year of 2010 and 2014, weighted and unweighted, respectively. Overall, a total of 4,741 and 4,505 diabetes adults aged 50 or older in 2010 and 2014 were included in the analyses, representing 18.8 million and 19.1 million older adults with diabetes respectively. The mean age of the diabetes patients was 1.9 years older in 2014 than that in 2010. Overall, diabetes patients dually covered by Medicare and Medicaid increased from $8 \%$ to $10 \%$ and the uninsured decreased from $6 \%$ to $4 \%$. Patients covered by private insurance also decreased from $30 \%$ to $25 \%$ based on weighted population estimates (Table 1 ).

Table 2 shows the prevalence rates of CRN by insurance status stratified by age between 50 and 64 and 65 or older. The overall CRN rates decreased from $27 \%$ to $21 \%$ and from $12 \%$ to $10 \%$ for those between 50 and 64 , and 65 or older, respectively, from 2010 to 2014. In general, there is a general trend of deceasing CRN rates across the insurance status by age strata.

Table 3 shows the adjusted odds ratio in the multivariate logit

Table 1 Socio-Economic and health characteristics, 2010 and 2014.

\begin{tabular}{|c|c|c|c|c|}
\hline & \multicolumn{2}{|c|}{ Sample Estimates } & \multicolumn{2}{|c|}{ Weighted Population Estimates } \\
\hline & 2014 & 2010 & 2014 & 2010 \\
\hline $\mathrm{N}$ with Diabetes (\% of total) & $4,505(25)$ & $4,741(23)$ & $19,058,793(23)$ & $18,853,729(21)$ \\
\hline \multicolumn{5}{|l|}{ Demographics } \\
\hline Mean Age N (SD) & $69.4(10)$ & $67.87(10)$ & $69.2(9)$ & $67.3(10)$ \\
\hline Male N (\%) & $1979(44)$ & $2155(45)$ & $9,187,045(48)$ & $9,278,668(49)$ \\
\hline \multicolumn{5}{|l|}{ Race } \\
\hline White $\mathrm{N}(\%)$ & $2886(64)$ & $3136(66)$ & $14,505,635(76)$ & $14,446,047(77)$ \\
\hline Black N (\%) & $1122(25)$ & $1134(24)$ & $2,724,426(14)$ & $2,723,629(14)$ \\
\hline Other N (\%) & $497(11)$ & $471(10)$ & $1,828,732(11)$ & $1,684,053(9)$ \\
\hline Hispanic N (\%) & $836(19)$ & $811(17)$ & $14,345,977(75)$ & $13,739,746(73)$ \\
\hline High School Degree N (\%) & $3121(69)$ & $3178(67)$ & $2,578,926(14)$ & $2,448,931(13)$ \\
\hline Income Median (Inter-quartile range) & $16,500(22,512)$ & $15,600(24,524)$ & $18,000(31,004)$ & $18,864(32,676)$ \\
\hline \multicolumn{5}{|l|}{ Insurance status } \\
\hline Medicare Only N (\%) & $2,528(56)$ & $2,558(54)$ & $10,607,737(56)$ & $9,443,756(50)$ \\
\hline Dual Eligibility N (\%) & $597(13)$ & $527(11)$ & $1,962,939(10)$ & $1,551,488(8)$ \\
\hline Medicaid Only N (\%) & $208(5)$ & $193(4)$ & $645,582(3)$ & $577,928(3)$ \\
\hline Private Insurance $\mathrm{N}(\%)$ & $893(20)$ & $1056(22)$ & $4,806,457(25)$ & $5,628,024(30)$ \\
\hline No Insurance $\mathrm{N}(\%)$ & $198(4)$ & $275(6)$ & $721,849(4)$ & $1,102,250(6)$ \\
\hline Other Public Insurance N (\%) & $81(2)$ & $132(3)$ & $314,224(2)$ & $550,283(3)$ \\
\hline \multicolumn{5}{|l|}{ Functional status } \\
\hline Activities of Daily Life N (s.d) & $1.91(1.83)$ & $1.80(1.75)$ & $1.68(1.68)$ & $1.65(1.62)$ \\
\hline Instrumental Activities of Daily Life N (s.d) & $1.44(1.30)$ & $1.40(1.24)$ & $1.25(1.18)$ & $1.25(1.12)$ \\
\hline \multicolumn{5}{|l|}{ Comorbid condition } \\
\hline Cancer N (\%) & $768(17)$ & $781(16)$ & $3,160,091(17)$ & $3,002,012(16)$ \\
\hline Depression N (\%) & $1437(32)$ & $1213(26)$ & $6,043,560(32)$ & $5,041,496(27)$ \\
\hline Heart Condition N (\%) & $1594(35)$ & $1662(35)$ & $6,578,843(35)$ & $6,330,011(34)$ \\
\hline Stroke N (\%) & $568(13)$ & $556(12)$ & $1,931,964(10)$ & $1,849,517(10)$ \\
\hline
\end{tabular}


Table 2 CRN rates by insurance status by age strata, 2010 and 2014 .

\begin{tabular}{|c|c|c|c|c|c|}
\hline \multirow[b]{2}{*}{ Age group } & \multirow[b]{2}{*}{ Insurance status } & \multicolumn{2}{|l|}{2014} & \multicolumn{2}{|l|}{2010} \\
\hline & & N (\%) Reporting CRN & P-Value & N Reporting CRN & P-Value \\
\hline \multirow{7}{*}{$50-64$} & Medicare w/o Medicaid & 411,151 (37) & \multirow{7}{*}{0} & $550,135(45)$ & \multirow{6}{*}{0} \\
\hline & Dual Eligible & $119,175(24)$ & & $181,979(35)$ & \\
\hline & Medicaid w/o Medicare & $173,275(29)$ & & $169,483(30)$ & \\
\hline & Private Insurance & $643,093(15)$ & & $1,016,649$ (19) & \\
\hline & No insurance & $175,808(26)$ & & 417,124 (39) & \\
\hline & Other Public Insurance & $51,180(19)$ & & $136,661(27)$ & \\
\hline & Overall for $50-64$ & $1,573,682(21)$ & & $2,472,031(27)$ & \\
\hline \multirow{7}{*}{65 or older } & Medicare w/o Medicaid & $939,124(10)$ & \multirow{6}{*}{0} & $955,624(12)$ & \multirow{6}{*}{0} \\
\hline & Dual Eligible & $141,419(10)$ & & $109,457(11)$ & \\
\hline & Medicaid w/o Medicare & $11,519(27)$ & & $0(0)$ & \\
\hline & Private Insurance & $39,570(10)$ & & $30,272(11)$ & \\
\hline & No insurance & $3,791(7)$ & & $9,651(23)$ & \\
\hline & Other Public Insurance & $1,452(3)$ & & $11,096(32)$ & \\
\hline & Overall for 65 or older & $1,136,875$ (10) & & $1,116,100(12)$ & \\
\hline
\end{tabular}

Legend: $p$-values were from chi-square tests between those reporting CRN and reporting no CRN during the year by age strata.

Table 3 Adjusted odds ratio of factors influencing CRN, 2010 and 2014.

\begin{tabular}{|c|c|c|c|c|c|c|}
\hline & \multicolumn{3}{|c|}{2014} & \multicolumn{3}{|c|}{2010} \\
\hline & Adjusted Odds Ratio & p-value & $95 \% \mathrm{Cl}$ & Adjusted Odds Ratio & p-value & $95 \% \mathrm{Cl}$ \\
\hline Age & 0.92 & 0.00 & $0.91,0.94$ & 0.91 & 0.00 & $0.89,0.92$ \\
\hline Gender (Female) & 1.35 & 0.05 & $1.00,1.82$ & 1.38 & 0.02 & $1.04,1.80$ \\
\hline Race (White) & referent & - & - & referent & - & - \\
\hline Race (Black) & 1.22 & 0.24 & $0.87,1.72$ & 1.32 & 0.08 & $0.96,1.82$ \\
\hline Race (Other) & 1.10 & 0.68 & $0.69,1.77$ & 1.27 & 0.30 & $0.81,2.00$ \\
\hline Hispanic & 0.71 & 0.10 & $0.46,1.07$ & 0.76 & 0.18 & $0.51,1.13$ \\
\hline High School Education & 0.89 & 0.44 & $0.65,1.20$ & 0.99 & 0.94 & $0.75,1.30$ \\
\hline Income (Log) & 0.87 & 0.07 & $0.75,1.01$ & 0.91 & 0.19 & $0.79,1.05$ \\
\hline Medicare only & referent & - & - & referent & - & - \\
\hline Dual Eligibility & 0.45 & 0.00 & $0.30,0.68$ & 0.64 & 0.02 & $0.44,0.93$ \\
\hline Medicaid only & 0.63 & 0.10 & $0.30,1.10$ & 0.27 & 0.00 & $0.16,0.46$ \\
\hline Private Insurance & 0.72 & 0.20 & $0.42,1.20$ & 0.63 & 0.04 & $0.41,0.98$ \\
\hline No Insurance & 1.17 & 0.66 & $0.58,2.33$ & 1.21 & 0.52 & $0.67,2.19$ \\
\hline Other Public Insurance & 0.84 & 0.72 & $0.32,2.33$ & 0.46 & 0.04 & $0.22,0.963$ \\
\hline ADL deficiencies & 1.08 & 0.08 & $1.00,1.18$ & 1.12 & 0.00 & $1.04,1.22$ \\
\hline IADL deficiencies & 0.96 & 0.55 & $0.85,1.09$ & 0.98 & 0.70 & $0.86,1.11$ \\
\hline Cancer & 0.82 & 0.31 & $0.56,1.19$ & 0.80 & 0.23 & $0.56,1.15$ \\
\hline Depression & 1.60 & 0.00 & $1.18,2.16$ & 1.50 & 0.00 & $1.14,1.98$ \\
\hline Heart Condition & 1.09 & 0.58 & $0.81,1.45$ & 1.23 & 0.12 & $0.94,1.61$ \\
\hline Stroke & 1.00 & 0.00 & $0.69,1.40$ & 0.73 & 0.07 & $0.51,1.03$ \\
\hline C statistics & \multicolumn{3}{|c|}{0.75} & \multicolumn{3}{|c|}{0.76} \\
\hline
\end{tabular}

Legend: Results from the multivariate logit regression analyses.

model of CRN rates for 2014 and 2010, respectively. Age is protective $(p<0.01)$, while female is associated with persistently higher CRN rates $(p=0.06, p<0.01)$ respectively. In terms of insurance coverage, dual eligibility is persistently protective. In addition, race (African American) became not statistically significant for variations in CRN in $2014(p=0.24)$.

\section{Conclusions}

Clearly, there is a change in the composition of insurance status among older diabetes patients in the U.S. between 2010 and 2014. More diabetes patients were eligible for both Medicare and Medicaid in 2014, reflecting the expansion of Medicaid under the ACA (and hence those previously with Medicare only became dually eligible). Since many diabetes patients are elderly or have disabilities which make them eligible for Medicare already prior to ACA, this shows a significant part of policy impact of the ACA in diabetes patients is gaining dual eligibility. The reduction in the number of uninsured is also likely attributable 
to the expansion of Medicaid under the ACA. Interestingly, fewer patients were insured by private insurance while the overall numbers of uninsured decreased, suggesting possible crowdingout of private insurance by driving patients into public insurance (Medicaid).

The overall impact of the change in composition of insurance status is a sharp reduction in CRN rates for those aged 50 to 64, and a modest decrease in CRN rates from 2010 to 2014 for those 65 years of older. This is because Medicaid only requires nominal out-of-pocket payments for medications, and also helps pay for the premium, deductible, and copayment of the Medicare program, and hence reduces the out-of-pocket payments by those patients, which in turn reduces CRN rates. This is an important policy effect of the ACA as it shows that the expansion of insurance coverage has an impact for reducing the access barrier in a measurable way. In the meantime, CRN rates among the diabetes patients ages 50 to 64 remain high despite a general decrease in CRN rates across the board. This is concerning because there is an inverse relationship between age of type 2 diabetes onset and complication risk and mortality, and the negative effect of diabetes on morbidity and mortality is greatest for those diagnosed at a young age [21]. The higher CRN rates in this age group means less effective treatment for those with more need and hence a greater loss in human productivity and life given the debilitating effect of diabetic progression. More research is much needed to develop interventions to reduce the cost burden in this subpopulation through insurance benefit design and coverage expansion.

This study shows that from 2010 to 2014, race became a less significant factor for variations in CRN. This is encouraging in that by expanding insurance coverage, one of the key factors that separate our society in access to care has been reduced to a significant degree. This means racial disparities can be addressed through expansion of insurance coverage and improvement of insurance design.

This study has broad implication for health and social policy. For instance, it suggests that insurance coverage plays a pivotal role in enabling patients to overcome their limited economic means and maintain access to much needed medical care even though their income is so low that they are in the Medicaid program. This is important because the role of health insurance is not only to ensure people against catastrophic events that are acute and life threatening, but also to help people maintain a life with dignity by accessing needed medical care to manage diseases that are chronically progressing. Furthermore, this study suggests that the expansion of insurance coverage such as the ACA's Medicaid expansion may decrease downstream spending in avoidable hospitalizations and emergency department visits by reducing CRN behaviors, meaning that expansion of the Medicaid program may help to pay for itself by reducing the downstream costs.

This study also has important implications for clinical practice. For example, research has found that a lack of communication about drug costs has been an important barrier to reducing CRN [22]. It is unclear if any patient-physician communication has happened on CRN particularly for those younger diabetes patients, and how and why there is a persistently high CRN rate among younger diabetes patients despite Medicare or MedicareMedicaid dual coverage. Given the heterogeneity of healthcare needs among diabetes patients between younger and older diabetes patients, it is imperative to understand that a more age-conscious approach can be developed to improve providerpatient communication and thereby reduce CRN.

Cost-related non-adherence to medical care is not unique to the United States. There is burgeoning body of research on cross-sectional comparison in CRN behaviors among developed countries. For example, in a study of adults aged 55 and older and living in the community in 11 developed countries, the authors found that following the lead of the USA with $16.8 \%$ in CRN to medication, Canada had the second highest national prevalence of CRN (8.3\%), followed by Australia $(6.8 \%)[23,24]$. Understanding the role of public insurance coverage expansion and its limitation can facilitate the discussion of how policy formulation can best serve the purpose of improving access to healthcare and wellbeing of millions people who struggle between the daily needs and medical care.

This study has a number of important limitations. First, the study only included those diabetes patients aged fifty years or older, and hence the conclusions may not be generalizable to those under 50 years of age. For example, we found that compared to the patients with Medicare only, the dual-eligible patients had lower CRN rates. Although it is encouraging that the additional Medicaid coverage of premiums, deductibles, and copayments may be helping reducing the CRN, it may not be sufficient for those with diabetes who are younger than 50 . Research has shown that if adults with diabetes of all age in the Medicare program were included, the CRN rates among the dual eligible were actually higher than those Medicare beneficiaries without Medicaid coverage in the Medicare program [25]. This shows the importance of age profile among diabetes patients and hence their behavioral differences. Second, this study is limited in that it is a cross-sectional study that pools data for two different years. While the progression of diabetes and its impact on CRN is beyond the scope of this study, a longitudinal study of the impact of the ACA may illuminate this relationship. Third, not all states adopted Medicaid expansion under ACA and 19 have not done so as of January 2017 [26]. We were not be able to compare directly the change in CRN by those states with expansion versus those without, but future research when such data become available should further ascertain the policy impacts of the ACA and its potential to influence patients behavior and reduce downstream costs.

In summary, we demonstrated a clear change in composition of insurance coverage among the older adults with diabetes after the implementation of the ACA. Such a change is accompanied by a general decrease in CRN rates across the insurance status, as the ACA moves more patients into Medicaid insurance coverage. In the meantime, racial disparities in the CRN rates appeared to have decreased. However, CRN rates remain high among patient's ages 50-60, despite a general reduction. This study contributes to the knowledge base of the importance of increasing insurance coverage and its influence on the patient's 
non-adherence behaviors due to costs. However, for younger patients with diabetes, the gap between knowledge and needed policy action seems to be large, and further research is much needed to develop interventions to reduce cost-related nonadherence to medical care, especially for those who are lowincome and economically vulnerable.

\section{Author Contributions}

Zhang conceptualized the study, conducted analyses, and prepared the manuscripts; Bhaumik prepared the data, conducted analysis; Huang critically reviewed the manuscript; Meltzer contributed to the manuscript development, and the obtaining of the funding support.

\section{References}

1 Sommers BD (2015) Health Care Reform's Unfinished Work. Remaining Barriers to Coverage and Access. N Engl J Med 373: 23952397.

2 Wilensky GR (2015) Improving and refining the Affordable Care Act. JAMA 314: 339-340.

3 Dorner SC, Jacobs DB, Sommers BD (2015) Adequacy of outpatient specialty care access in marketplace plans under the Affordable Care Act. JAMA 314: 1749-1750.

4 Madden JM, Graves AJ, Zhang F, Adams AS, Briesacher BA, et al. (2008) Cost-related medication nonadherence and spending on basic needs following implementation of Medicare Part D. JAMA 299: 1922-1928.

5 Naci H, Soumerai SB, Ross-Degnan D, Zhang F, Briesacher BA, et al. (2014) Medication affordability gains following Medicare Part $D$ are eroding among elderly with multiple chronic conditions. Health Aff 33: $1435-1443$.

6 Briesacher BA, Gurwitz JH, Soumerai SB (2007) Patients at-risk for cost-related medication nonadherence: a review of literature. J Gen Int Med 22: 864-871.

7 Goldman DP, Joyce GF, Zheng Y (2007) Prescription drug cost sharing: associations with medication and medical utilization and spending and health. JAMA 298: 61-69.

8 Tamblyn R, Laprise R, Hanley JA, Abrahamowicz M, Scott S, et al. (2001) Adverse events associated with prescription drug cost-sharing among poor and elderly persons. JAMA 285: 421-429.

9 Mojtabai R, Olfson M (2003) Medication costs, adherence, and health outcomes among Medicare beneficiaries. Health Aff 22: 220-229.

10 Kurlander JE, Kerr EA, Krein S, Heisler M, Piette JD (2009) Cost-related nonadherence to medications among patients with diabetes and chronic pain: factors beyond finances. Diabetes Care 2: 2143-2148.

11 Aikens JE, Piette JD (2009) Diabetic patients' medication underuse, illness outcomes and beliefs about antihyperglycemic and antihypertensive treatments. Diabetes Care 32: 19-24.

12 Piette JD, Wagner TH, Potter MB, Schillinger D (2004) Health insurance status, cost-related medication underuse, and outcomes among diabetes patients in three systems of care. Med Care 42: 102-109.

\section{Funding source}

This study is supported in part by Chicago Center for Diabetes Translation Research (CCDTR) Pilot and Feasibility Grant (Zhang \& Meltzer) (P30 DK092949), and NIH 1R21AG053749-01 (Meltzer \& Zhang).

\section{Conflicts of Interest}

The authors have no conflicts of interest. Zhang has an ownership interest in Chicago MedInfo Group, LLC.

13 Blumenthal D, Melinda A, Rachel N (2015) The Affordable Care Act at 5 Years. New England Journal of Medicine 372: 2451-2458.

14 The Health and Retirement Study. Growing Older in America: The Health and Retirement Study.

15 Manning WG, Mullahy J (2001) Estimating log models: to transform or not to transform? J Health Econ 20: 461-494.

16 Zhang JX, Lee JU, Meltzer DO (2015) The Effect of Functional Limitations and Hospitalization on Out-of-Pocket Medical Payments in older Adults. Ann Community Med Pract 1: 1004.

17 Bambauer KZ, Safran DG, Ross-Degnan D, Zhang F, Adams AS, et al. (2007) Depression and cost-related medication nonadherence in Medicare beneficiaries. Arch Gen Psychiatry 64: 602-608.

18 Giovannucci E, Harlan DM, Archer MC, Bergenstal RM, Gapstur SM, et al. (2010) Diabetes Care 33: 1674-1685.

19 Zhang JX, Huang ES, Drum ML, Kirchhoff AC, Schlichting JA, et al. (2009) Insurance status and quality of diabetes care in community health centers. Am J Public Health 99: 742-747.

20 Pencina MJ, D'Agostino RB (2015) Evaluating Discrimination of Risk Prediction Models: The C Statistic. JAMA 314: 1063-1064.

21 Al-Saeed AH, Constantino MI, Molyneaux L, D'Souza M, Gisler FL, et al. (2016) An Inverse Relationship Between Age of Type 2 Diabetes Onset and Complication Risk and Mortality: The Impact of YouthOnset Type 2 Diabetes. Diabetes Care 39: 823-829.

22 Alexander GC, Casalino LP, Tseng CW, McFadden D, Meltzer DO (2004) Barriers to patient-physician communication about out-ofpocket costs. J Gen Intern Med 19: 856-860.

23 Zhang JX (2017) Is Cost-Related Non-Adherence to Medical Care Unique to the USA? J Gerontol Geriatr Res 6: e146.

24 Morgan SG, Lee A (2017) Cost-related non-adherence to prescribed medicines among older adults: a cross-sectional analysis of a survey in 11 developed countries. BMJ Open 7: e014287.

25 Zhang JX, Meltzer DO (2016) The High Cost-related Medication Nonadherence Rate among Medicare-Medicaid Dual-Eligible Diabetes Patients. J Health Med Econ 2 pii: 13.

26 The Henry J Kaiser Family Foundation (2018) Status of State Action on the Medicaid Expansion Decision. 